\title{
Effect of Different Herbal Seed Powders as Feed Additives on Growth Performance, Haematology, Digestive Enzyme Activity and Stress Levels of GIFT Tilapia (Oreochromis niloticus) Fingerlings
}

Irtiqa Hamdani ( $\square$ irtiqahamdani38@gmail.com )

Tamil Nadu Fisheries University https://orcid.org/0000-0002-8134-546X

Cheryl Antony

Fisheries College and Research Institute

Research Article

Keywords: Celery, Fenugreek, Coriander, GIFT Tilapia, Growth, Haematology, Enzymes

Posted Date: March 7th, 2022

DOI: https://doi.org/10.21203/rs.3.rs-1397825/v1

License: @ (i) This work is licensed under a Creative Commons Attribution 4.0 International License.

Read Full License 


\section{Abstract}

Present research study was conducted for a period of 60 days to study the effects of different herbal seed powders - Celery (Apium graveolens), Fenugreek (Trigonella foenum-graecum) and Coriander (Coriandrum sativum) as feed additives on growth, haematology, digestive enzyme activity and stress levels of GIFT Tilapia (Oreochromis niloticus) fingerlings. Ten experimental diets were formulated, nine diets using Celery, Fenugreek and Coriander seed powder incorporated at 1.5\%, 2\% and 2.5\% levels each and one control diet with no supplement. Protein content of the formulated diets ranged from 27.95 to $29.93 \%$ while as the lipid content ranged from 8.1 to $9.3 \%$. The study design comprised of thirty PVC tanks (50L capacity) for triplicate experiment design with ten treatment groups per replicate. A total of three hundred fingerlings with ten fingerlings per tank were reared. At the commencement of the experiment, the fingerlings measured $3.48 \pm 0.46 \mathrm{~cm}$ and weighed $3.41 \pm 0.43 \mathrm{~g}$ on average. The fingerlings were reared in an indoor wet laboratory with filtered borewell water as freshwater source. Fingerlings were fed at the rate of $5 \%$ body weight daily in three rations. Growth and blood sampling was done fortnightly while as digestive enzyme activity and stress parameters were analysed at the end of the experiment. By the end of the experiment, growth performance in terms of Weight Gain, Specific Growth Rate (SGR), Relative Growth Rate (RGR), Protein Efficiency Ratio (PER), Feed Conversion Efficiency (FCE) and Feed Conversion Rate (FCR) was recorded. Treatment group fed with $2 \%$ Coriander seed powder supplemented diet showed the best results among all treatment groups. Highest Hepato-Somatic Indices (HSI) and Viscero-Somatic Indices (VSI) were observed in treatment group fed with 2.5\% Fenugreek supplemented diet. Among the haematological indices, highest count of Red Blood Cells (RBC) was recorded in treatment group fed with 2.5\% Coriander supplemented diet. Other parameters such as White Blood Cells (WBC), Haemoglobin (Hb), Haematocrit (Hct), Mean Corpuscular Haemoglobin ( $\mathrm{MCH})$ and Mean Corpuscular Haemoglobin Concentration $(\mathrm{MCHC})$ values were recorded the maximum in fish treatment group fed with $2 \%$ Coriander supplemented diets. Maximum Mean Cell Volume (MCV) value was seen in Celery seed supplemented diets at $2 \%$ level. Highest blood protein content and glucose levels were observed in Coriander incorporated diets $(1.5 \%)$ and control respectively. Further, among the digestive enzymes activity recorded, highest Trypsin activity was recorded in treatment group fed with Celery supplemented diet (2\%), Lipase in Celery supplemented diet (1.5\%) and Amylase in Fenugreek supplemented diet (2.5\%). The oxidative enzymes were recorded for the estimation of stress. All the parameters (Catalase, Lipase Dehydrogenase (LDH), Malate Dehydrogenase (MDH), Sodium Oxide Dismutase (SOD) and Nitro-Blue Tetrazolium (NBT) showed the maximum activity in the group treated with Coriander seed supplemented diet at $2.5 \%$ level. By the end of the experiment trial, fingerlings fed with different seed incorporated diets showed a visible change in their body colouration. The results obtained from the present study suggest that supplementing fish diets with different herbal seeds reveal pronounced effects on different functional parameters such as growth, haematology, digestive and oxidative enzymes activity.

\section{Introduction}

Aquaculture is one of the fastest growing food producing sectors, with the highest potential to assist marine and inland capture fisheries to meet the global demand for aquatic food (FAO, 2016). Global 
aquaculture production is continuously increasing at an intensely rapid pace. Among the freshwater cultured fish species of the world, tilapias are reported to be the second most important group of farmed fin fish just after carps (El-Sayed A. F. M., 2019). Tilapia is one of the significant candidate species in aquaculture due to its unique favorable characteristics like rapid growth, high survival rate, stress and disease resistance and wide range feed acceptance (El-Sayed, A.F.M., 2006).

In India, Tilapia is being intensively cultured for these traits. Under the Marine Products Exports Development Authority (MPEDA), the Government of India has promoted the culture of Genetically Improved Farmed Tilapia (GIFT), Oreochromis niloticus, through Rajiv Gandhi Center for Aquaculture (RGCA) (Behera B.K. et al., 2018).

However, improving fish performance and developing disease resistance of cultured Tilapia fishes becomes one of the major challenges in aquaculture industry (Nicolae et. al., 2016). In this regard, medicinal plants are rapidly evolving as an alternative approach for controlling diseases in aquaculture (Awad and Awaad, 2017; Hoseini et. al., 2019; Citarasu et. al., 2010). Medicinal plants comprise of herbs, seaweeds, herbal extracts, spices, plant derived compounds and traditional Chinese herbs (Van Hai, 2015). The use of medicinal herbs has proven to be a suitable alternative to antibiotics (Abarike et. al., 2018; Awad and Awaad, 2017), to boost fish immunity (Van Hai, 2015) and as feed additives in aquaculture (Chang 2000). Further, they are environment friendly and cost effective with almost no side effects (Jian and $\mathrm{Wu}, 2004)$. Celery, Fenugreek and Coriander are such examples to name a few.

Celery (Apium graveolens) is a member of the Apiaceae family native to South America, South Europe, Africa and Asia (Sowbhagya, 2014). Its medicinal properties can be derived from different parts such as fresh herb, stalk, leaves, seeds, seed oil and oleoresins (Sowbhagya, 2014). Celery is quiet rich in phytochemically active constituents such as phenols, flavonoids and essential oils that are derived from its roots, seeds and leaves (Aydemir and Becerik, 2011; Li et al., 2014), Studies have revealed its antiinflammatory, hepatoprotective, (Ahmed et al., 2002), antioxidant (Li et al., 2014), anti-bacterial, anticancer, anti-hepatotoxic, anti-hypercholesterolemic, analgesic and anti-spasmodic (Modaresi et al., 2012) properties.

Fenugreek (Trigonella foenum-graecum L.), a leguminous plant belonging to family Leguminosae, is widely cultivated in the Mediterranean, Western Asia, North America and Northern India (Mehboob et al., 2017). The seeds of this herb are high in protein and an important source of diosgenin, a hormonal precursor (Abdelwahab \& El-Bahr, 2012). Studies have revealed the antioxidant (Kaviarasan et. al., 2004) and immunostimulatory (Sayeed et. al., 2003) properties of fenugreek.

Coriander (Coriandrum sativum) is an annual herb of the Apiaceae or carrot family. Also known by the name Cilantro, this herb is native to South Eastern Europe but grows all over Europe, Middle East, China, Turkey and India (Laribi B. et. al., 2015; Al-Mofleh et al., 2006). Coriander seeds are quiet high in active compounds (Lasram et al., 2019; Begnami et. al., 2018). Coriander seeds are also rich in Linalool (40$50 \%$ ), a terpenoid having antimicrobial, anti-inflammatory and strong antioxidant properties (Farsani et al., 2019). 
The present experiment was undertaken to study the effects of the above mentioned three medicinal herbs - Celery, Fenugreek and Coriander seed powder at different levels as feed additives in GIFT Tilapia diets to study for its effect on growth, haematological indices, digestive enzyme profile and oxidative stress.

\section{Materials And Methods}

\subsection{Experimental setup}

GIFT Tilapia fingerlings were procured from GIFT Tilapia hatchery at Madurai, Tamil Nadu. On the commencement of the experiment, all the fishes were measured and weighed individually and an average total length and weight of $3.48 \pm 0.46 \mathrm{~cm}$ and $3.41 \pm 0.43 \mathrm{~g}$ respectively was recorded. A total of 300 fingerlings were equally distributed into three groups (Triplicate design) and each group (100 fingerlings) was further divided into 10 treatment groups ( 9 supplemented diet and 1 control diet fed group) with each treatment group maintained at 10 fingerlings per tank. Totally $30 \mathrm{PVC}$ tanks of $50 \mathrm{~L}$ capacity each were used. The tanks were supplied with filtered bore-well water and adequate oxygen supply was ensured. Siphoning of wastes was done once daily followed by $10 \%$ water exchange. Details of experimental codes used for the experiment are given in Table 1.

Table 1. Details of Experiment Codes used in the present study

\begin{tabular}{lll} 
Code & Treatment & Replicate Codes \\
\hline T1 & Celery seed diet (1.5\%) & T1R1, T1R2, T1R3 \\
\hline T2 & Celery seed diet (2.0\%) & T2R1, T2R2, T2R3 \\
\hline T3 & Celery seed diet (2.5\%) & T3R1, T3R2, T3R3 \\
\hline T4 & Fenugreek seed diet (1.5\%) & T4R1, T4R2, T4R3 \\
\hline T5 & Fenugreek seed diet (2.0\%) & T5R1, T5R2, T5R3 \\
\hline T6 & Fenugreek seed diet (2.5\%) & T6R1, T6R2, T6R3 \\
\hline T7 & Coriander seed diet (1.5\%) & T7R1, T7R2, T7R3 \\
\hline T8 & Coriander seed diet (2.0\%) & T8R1, T8R2, T8R3 \\
\hline T9 & Coriander seed diet (2.5\%) & T9R1, T9R2, T9R3 \\
\hline Control & Basal diet & C1, C2, C3
\end{tabular}

\subsection{Feed preparation and administration}

Ten different experiment diets were formulated using the feed ingredients as listed in Table 2. Nine out of ten diets were prepared by incorporating experimental ingredients, Celery (Apium graveolens), Fenugreek (Trigonella foenum-graecum) and Coriander (Coriandrum sativum) seed powder at 1.5, 2.0 and $2.5 \%$ levels 
each. One out of ten diets was the control diet prepared only from the basal ingredients. The experimental ingredients were ground to fine powder before incorporating into the diet. The incorporation levels of different ingredients are presented in Table 2. The main source of protein in all the diets was fish meal and soybean meal. The proximate composition of the formulated diets is given in Table 3 .

All the ingredients were thoroughly mixed into soft dough and extruded using a hand pelletizer. The pelleted feed was air dried for 6-8 hours, crumbled and stored at room temperature in air-proof containers.

GIFT Tilapia fingerlings were manually fed daily at the rate of $5 \%$ body weight for a period of 60 days. The daily feed ration was roughly divided and fed three times per day. It was made sure that the next ration of the daily feed was given only when the prior ration was fully consumed and nothing was left uneaten. The feed supply was adjusted at $5 \%$ to ensure that the daily ration remained a fixed proportion of the body weight as time progressed and the fish increased in size as recorded by sampling. The water quality parameters like temperature, $\mathrm{pH}$, salinity, alkalinity, ammonia, nitrogen etc. were all recorded according to APHA (1998) and found within acceptable ranges throughout the experimental period (Boyd, 1982). Average values of water quality parameters recorded during the study are summarized in Table 3.

\subsection{Proximate Composition of formulated diets}

Individual sample from each treatment group was subjected to proximate composition analysis. Kjeldahl's method (Kirk, 1950) was used to determine the Crude protein content (KelPlus - KelFlow digestion unit). Lipid content was determined by Folch method (Folch et al., 1957). Moisture and Fibre content was estimated by the method of AOAC, 2016. 
Table 2. Incorporation level of different feed ingredient in formulated feeds

\begin{tabular}{|c|c|c|c|c|c|c|c|c|c|c|c|c|}
\hline \multirow[t]{2}{*}{ Ingredient } & \multicolumn{12}{|c|}{ Percentage inclusion level of feed ingredients } \\
\hline & & & & 2 & T3 & 4 & T5 I & T6 & & T8 & T9 & Control \\
\hline Fish Meal & 22 & 22 & 22 & 22 & 22 & 22 & 22 & 22 & 22 & 22 & & \\
\hline Soybean Meal & 20 & 20 & 20 & 20 & 20 & 20 & 20 & 20 & 20 & 20 & & \\
\hline Corn Flour & 18 & 18 & 18 & 18 & 18 & 18 & 18 & 18 & 18 & 18 & & \\
\hline Corn Gluten & 8 & 8 & 8 & 8 & 8 & 8 & 8 & 8 & 8 & 8 & & \\
\hline Cassava Starch & 5 & 5 & 5 & 5 & 5 & 5 & 5 & 5 & 5 & 5 & & \\
\hline Wheat & 21.5 & 21 & 20.5 & 21.5 & 21 & 20.5 & 21.5 & $5 \quad 21$ & 20.5 & $5 \quad 23$ & & \\
\hline Calcium* & 2 & 2 & 2 & 2 & 2 & 2 & 2 & 2 & 2 & 2 & & \\
\hline Vitamin Premix $* *$ & 1 & 1 & 1 & 1 & 1 & 1 & 1 & 1 & 1 & 1 & & \\
\hline Mineral Premix** & 1 & 1 & 1 & 1 & 1 & 1 & 1 & 1 & 1 & 1 & & \\
\hline Celery Seed Powder & 1.5 & 2.0 & 2.5 & - & - & - & - & - & - & - & & \\
\hline Fenugreek Seed Powder & - & - & - & 1.5 & 2.0 & 2.5 & - & - & - & - & & \\
\hline Cori & - & - & - & - & - & - & 1.5 & 2.0 & 2.5 & - & & \\
\hline
\end{tabular}

*Dicalcium Phosphate (47\% Calcium, $589 \mathrm{mg} / \mathrm{g})$

$*{ }^{*}$ Growel, chelated vitamin-mineral mixture; Each $1 \mathrm{~kg}$ contains $8 \times 10^{5} \mathrm{IU}$ Vit.A, $8 \times 10^{4} \mathrm{IU}$ D, $680 \mathrm{mg}$ Vit.E, $1200 \mathrm{mg}$ Nicotinamide, $2200 \mathrm{mg}$ Cobalt, $4700 \mathrm{mg}$ Copper, $600 \mathrm{mg}$ Iodine, $2200 \mathrm{mg}$ Iron, 6500mg Magnesium, 3300mg Manganese, 200mg Potassium, 40mg Sodium, $0.95 \%$ Sulphur, $10000 \mathrm{mg}$ Zinc, 30\% Calcium and $15 \%$ Phosphorous

Table 3. Proximate Composition of formulated experimental diets

Proximate Parameter (\%)

\begin{tabular}{clcccc} 
Experiment diet & Crude Protein & $\begin{array}{c}\text { Moisture } \\
\text { (\%Min) }\end{array}$ & $\begin{array}{c}\text { Lipid Content } \\
\text { (\%) }\end{array}$ & $\begin{array}{c}\text { Crude Fibre } \\
\text { (\%Min) }\end{array}$ & (\%Max) \\
\hline T1 & 28.74 & 5.72 & 8.3 & 5.01 \\
T2 & 28.71 & 6.10 & 8.1 & 4.99 \\
T3 & 28.56 & 6.30 & 8.2 & 5.13 \\
T4 & 29.93 & 5.02 & 9.3 & 4.67 \\
T5 & 29.90 & 5.10 & 9.1 & 4.77 \\
T6 & 29.80 & 4.99 & 9.2 & 4.56 \\
T7 & 29.15 & 6.51 & 8.9 & 4.15 \\
T5 & 29.52 & 6.42 & 9.1 & 4.13 \\
T9 & 29.75 & 6.39 & 9.3 & 4.23 \\
Control & 27.95 & 6.31 & 9.3 & 5.05 \\
\hline
\end{tabular}

Table 4. Water Quality Parameters recorded during the experiment 


\begin{tabular}{ll} 
Water quality parameter & Mean values* \\
\hline Temperature $(\mathbb{} \mathrm{C})$ & $28.12 \pm 0.56$ \\
\hline $\mathrm{pH}$ & $7.6 \pm 0.04$ \\
\hline Carbon Dioxide $(\mathrm{mg} / \mathrm{L})$ & Absent \\
\hline Dissolved Oxygen $(\mathrm{mg} / \mathrm{L})$ & $5.13 \pm 0.34$ \\
\hline Ammonia $(\mathrm{mg} / \mathrm{L})$ & $0.0044 \pm 0.0011$ \\
\hline Nitrite $(\mathrm{mg} / \mathrm{L})$ & $0.042 \pm 0.0043$ \\
\hline Nitrate $(\mathrm{mg} / \mathrm{L})$ & $0.43 \pm 0.06$ \\
\hline Alkalinity $(\mathrm{mg} / \mathrm{L})$ & $95.63 \pm 0.21$ \\
\hline Calcium $(\mathrm{mg} / \mathrm{L})$ & $74.02 \pm 0.96$ \\
\hline Magnesium (mg/L) & $21.12 \pm 1.003$ \\
\hline Hardness $(\mathrm{mg} / \mathrm{L})$ & $470.254 \pm 11.27$ \\
\hline Phosphate $(\mathrm{mg} / \mathrm{L})$ & $0.041 \pm 0.0037$ \\
\hline Chloride $(\mathrm{mg} / \mathrm{L})$ & $118.127 \pm 5.776$ \\
\hline Total Suspended Solids & $44.15 \pm 0.841$ \\
\hline Total Dissolved Solids & $42.83 \pm 1.03$ \\
\hline
\end{tabular}

*Data expressed as Mean \pm Standard Deviation

\subsection{Growth Sampling}

Sampling for length and weight measurement was carried out at fortnightly intervals on day 0 , day 15 , day 30 , day 45 and day 60 of the experimental period. Length (in $\mathrm{cm}$ ) was measured using a geometric scale and weight (in g) was recorded using a potable weighing balance. Growth parameters were calculated according to Silva and Anderson (1994). Five fishes from each tank were selected randomly and individually weighed for growth analysis. Somatic indices \{Hepato-somatic Index (HSI) and Viscerosomatic Index (VSI)\} were measured as per Zheng et al., (2009). Three random fishes from each tank were sampled. Liver and viscera from each fish was carefully removed and weighed for calculating HSI and VSI respectively. The calculations for growth analysis are given in section 2.8.

\subsection{Haematological Analysis}

Blood sampling was carried out on day 0 , day 15 , day 30 , day 45 and on day 60 . Fishes were starved for about 24 hours prior to collection of blood. Two random fishes from each treatment group were taken for 
blood collection. Blood was drawn from the caudal vein of the fish using $1 \mathrm{ml}$ sterile disposable plastic insulin syringe with a needle size of $0.25 \mathrm{~mm}$. The anticoagulant used for haematological analysis in present study was EDTA (Ethylene Di-amine Tetra-Acetic acid). The dispo-syringe was lightly coated with EDTA solution prior to drawing of blood. The collected blood was transferred to EDTA coated vials ( $2 \mathrm{ml}$ ) and kept in ice. Collected blood samples were immediately subjected to analysis.

The Red Blood Cell (RBC) and White Blood Cell (WBC) counts were determined using Neubauer haemocytometer (Blaxhall and Daisley, 1973; Rusia and Sood, 1992). Haemoglobin (Hb) concentrations were determined using Cyanomethaemoglobin method as described by Drabkin (1946). Haematocrit values were determined by Microhaematocrit method described by Nelson and Morris (1989). Blood indices like Mean Cell Volume (MCV), Mean Cell Haemoglobin (MCH), Mean Cell Haemoglobin Concentration (MCHC) were assessed according to the equations of Wintrobe, (1934). Serum indices like Glucose were estimated by the method of Trinder (1969) and Albumin, Globulin and Total Protein was calculated by the method described by Lowry et al. (1951).

\subsection{Digestive Enzymes Analysis}

At the end of the experiment, two random fishes from each treatment group were dissected. For the preparation of tissue homogenate, intestinal part of the digestive tract was carefully removed and immediately transferred on to ice. The extracted specimen was gently rinsed with cold tris-buffer $\mathrm{HCl}$. Excessive fat tissue was carefully removed using forceps followed by weighing the sample. The sample was homogenized using hand-held homogenizer and stored in icebox $\left(4-5^{\bullet} \mathrm{C}\right)$. The homogenate was centrifuged at 5000 RPM for $10 \mathrm{~min}$ at $4^{\bullet} \mathrm{C}$ in a cooling centrifuge. After centrifugation, the supernatant was removed, transferred and stored in a $15 \mathrm{ml}$ sterile vial tubes. The samples were stored at $-80^{\bullet} \mathrm{C}$ for further analysis.

Estimation of Amylase was done according to Somogyi-Nelson method (Somogyi, 1952) using starch as substrate. Estimation of Lipase was done by the method described by Shihabi and Bishop (1971) using olive oil as substrate. Estimation of Trypsin was carried out by the method described by White and Bowman (1947) using casein as substrate.

\subsection{Stress Analysis}

At the end of the experiment, two random fishes from each treatment group were sampled for the estimation of oxidative enzymes to analyze the stress levels in fishes from each treatment group. Enzymes were analysed in liver, muscle and gill of fish from each treatment group. For the preparation of tissue homogenate, tissues (liver, gills and muscle) were homogenized with $0.25 \mathrm{M}$ sucrose solution on wet basis $(\mathrm{pH} 7.0,1: 20 \mathrm{~W} / \mathrm{V})$ in a handheld homogenizer. The samples were stored in icebox $\left(4-5^{\bullet} \mathrm{C}\right)$. The homogenate was centrifuged at 5000 RPM for 10 min at $4{ }^{\circ} \mathrm{C}$ in a refrigerated centrifuge. After centrifugation, the supernatant was removed, transferred and stored in a $15 \mathrm{ml}$ sterile vial tubes. The samples were stored at $-20^{\bullet} \mathrm{C}$ for further analysis. 
Stress levels in treated fishes were estimated by Catalase, Lactate Dehydrogenase (LDH), Malate Dehydrogenase (MDH), Superoxide Dismutase (SOD) and NitroBlue Tetrazolium (NBT) assays. Catalase activity (CAT) was estimated according to the method of Takahara et al. (1960). The LDH activity was measured according to the modified method of Liu et. al.,(1981). The MDH activity was measured according to the method of Bernstein and Grisham (1978). The Superoxide Dismutase (SOD) activity was estimated by the modified method of Misra and Fridrovich (1972). Respiratory burst activity of the phagocytes was measured by NitroBlue Tetrazolium (NBT) assay according to the modified method of Anderson and Siwiki (1995).

\subsection{Calculations and Statistical Analysis}

- Food Conversion Ratio (FCR) = Total feed $(\mathrm{g}) /$ Weight gain $(\mathrm{g})$

- Protein Efficiency Ratio (PER) = Weight Gain / Protein Intake

- Food Conversion Efficiency $(\mathrm{FCE})=$ Net Biomass $/$ Total Feed Given $* 100$

- Biomass = Average Body Weight $x$ No. of fishes

- Net Biomass Production = Final Biomass Obtained - Initial Biomass Stocked

- Specific Growth Rate (SGR) = (Ln Final Weight - Ln Initial Weight $)$ * 100 / Days

- Average Daily Weight Gain (ADWG) = Final Weight - Initial Weight $/$ DOC

- Daily Weight Gain $(\%)=$ ADWG / Initial Weight of fishes * 100

- Total Weight Gain (TWG) = (Mean Final Weight - Mean Initial Weight)

- Relative Growth Rate (Length) $(R G R-L)=\left(L_{2}-L_{1}\right) / L_{1} * 100$

- Relative Growth Rate (Weight) $(\mathrm{RGR}-\mathrm{W})=\left(\mathrm{W}_{2}-\mathrm{W}_{1}\right) / \mathrm{W}_{1} * 100$

- Hepato-Somatic Index (HSI) = Weight of Liver (g) / Weight of Fish (g) * 100

- Viscero-Somatic Index (VSI) = Weight of Viscera (g) / Weight of Fish (g) * 100

- Condition Factor $(\mathrm{K})=$ Body Weight $(\mathrm{g}) /\{\text { Body Length }(\mathrm{cm})\}^{3} * 100$

- Survival Rate $(S R)=$ No. of Fishes Survived $/$ No of Fishes Stocked $* 100$

$\left[\mathrm{L}_{1}=\right.$ Initial Length; $\mathrm{L}_{2}=$ Final Length; $\mathrm{W}_{1}=$ Initial Weight $; \mathrm{W}_{2}=$ Final Weight $\mathrm{t}_{1}=$ Initial DOC; $\mathrm{t}_{2}=$ Final DOC $]$

All the data obtained during experiment was statistically analyzed and expressed as mean \pm standard deviation and the data variables were checked for homogeneity. All the parameters were analyzed by one way analysis of variance (ANOVA) followed by Duncan's multiple range tests to determine the significant differences between the means using IBM-SPSS statistics version 20. The p-values of $<0.05$ and $<0.01$ were considered to represent the significance level between treatment groups.

\section{Results}

\subsection{Growth performance and feed utilization}


Incorporation of different herbal seeds at different levels significantly affected the growth performance and feed conversion of GIFT Tilapia. Survival Rate (SR) of fish fed with experiment diets showed better performance than control diet fed group. 100\% survival rate was recorded in T4, T5, T6 and T9 and lowest in control (75\%). Feed ration was equally quantified for all treatment groups, however the lowest FCR was observed in T8 (1.26 \pm 0.02$)$ compared to other treatment groups and control. Highest Weight gain, Daily weight gain, Biomass, Specific Growth Rate, Relative Growth Rate, Protein Efficiency Ratio and Feed Conversion Efficiency and lowest Feed Conversion Ratio was recorded in T8 (Table 4\&5).Data recorded was found to be statistically significant $(p<0.05)$.

Table 5. Growth parameters recorded during the experiment - I

\begin{tabular}{|c|c|c|c|c|c|c|}
\hline Treatment & WG & DWG & Biomass & SGR & RGR & PER \\
\hline T1 & $4.91 \pm 0.20^{\mathrm{a}}$ & $2.38 \pm 0.05^{a}$ & $49.26 \pm 2.03^{a}$ & $1.48 \pm 0.02^{b}$ & $142.22 \pm 2.23^{b}$ & $\begin{array}{l}1.66 \pm \\
0.06^{\mathrm{b}}\end{array}$ \\
\hline T2 & $5.10 \pm 0.17^{b}$ & $2.48 \pm 0.13^{a}$ & $51.11 \pm 1.73^{b}$ & $1.52 \pm 0.05^{c}$ & $149.17 \pm 7.54^{b}$ & $\begin{array}{l}1.72 \pm \\
0.05^{\mathrm{c}}\end{array}$ \\
\hline T3 & $4.22 \pm 0.11^{\mathrm{a}}$ & $2.05 \pm 0.05^{a}$ & $42.31 \pm 1.08^{a}$ & $1.34 \pm 0.02^{\mathrm{a}}$ & $123.37 \pm 3.55^{a}$ & $\begin{array}{l}1.43 \pm \\
0.04^{\mathrm{a}}\end{array}$ \\
\hline T4 & $6.35 \pm 0.23^{c}$ & $3.09 \pm 0.15^{b}$ & $63.61 \pm 2.42^{c}$ & $1.75 \pm 0.05^{\mathrm{e}}$ & $185.67 \pm 9.58^{d}$ & $\begin{array}{l}2.14 \pm \\
0.08^{\mathrm{e}}\end{array}$ \\
\hline T5 & $4.43 \pm 0.14^{\mathrm{a}}$ & $2.11 \pm 0.06^{a}$ & $44.37 \pm 1.48^{a}$ & $1.37 \pm 0.02^{\mathrm{a}}$ & $127.15 \pm 3.86^{\mathrm{a}}$ & $\begin{array}{l}1.49 \pm \\
0.05^{a}\end{array}$ \\
\hline T6 & $5.60 \pm 0.30^{b}$ & $2.75 \pm 0.22^{a}$ & $56.07 \pm 3.05^{b}$ & $1.62 \pm 0.08^{d}$ & $165.35 \pm 13.43^{c}$ & $\begin{array}{l}1.89 \pm \\
0.10^{d}\end{array}$ \\
\hline T7 & $7.66 \pm 0.20^{d}$ & $3.74 \pm 0.19^{b}$ & $76.71 \pm 2.11^{d}$ & $1.96 \pm 0.06^{f}$ & $224.51 \pm 11.91^{\mathrm{e}}$ & $\begin{array}{l}2.59 \pm \\
0.07^{\mathrm{e}}\end{array}$ \\
\hline T8 & $8.10 \pm 0.10^{e}$ & $4.00 \pm 0.04^{c}$ & $81.01 \pm 1.49^{e}$ & $\begin{array}{l}2.04 \pm \\
0.01^{g}\end{array}$ & $240.34 \pm 2.59^{e}$ & $\begin{array}{l}2.74 \pm \\
0.05^{\mathrm{e}}\end{array}$ \\
\hline T9 & $6.08 \pm 0.32^{c}$ & $3.01 \pm 0.02^{b}$ & $60.29 \pm 3.64^{c}$ & $1.72 \pm 0.01^{\mathrm{e}}$ & $180.72 \pm 1.26^{d}$ & $\begin{array}{l}2.74 \pm \\
0.05^{\mathrm{e}}\end{array}$ \\
\hline Control & $5.11 \pm 0.49^{b}$ & $2.69 \pm 0.21^{a}$ & $53.62 \pm 2.17^{b}$ & $1.60 \pm 0.08^{d}$ & $161.54 \pm 12.90^{c}$ & $\begin{array}{l}2.04 \pm \\
0.12^{\mathrm{e}}\end{array}$ \\
\hline
\end{tabular}

Data expressed as Mean \pm Standard deviation.

Values with different superscripts within column differ significantly $(p<0.05)$

Table 6. Growth parameters recorded during the experiment - II 


\begin{tabular}{|c|c|c|c|c|c|c|}
\hline Treatment & FCE & FCR & HSI & VSI & SR & K \\
\hline $\mathrm{T} 1$ & $48.29 \pm 1.99^{a}$ & $2.07 \pm 0.08^{e}$ & $0.63 \pm 0.09^{b}$ & $8.19 \pm 0.34^{c}$ & $\begin{array}{l}95 \pm \\
0.70^{b}\end{array}$ & $\begin{array}{l}1.05 \pm \\
0.07^{\mathrm{b}}\end{array}$ \\
\hline $\mathrm{T} 2$ & $50.09 \pm 1.69^{b}$ & $1.99 \pm 0.06^{d}$ & $0.66 \pm 0.03^{b}$ & $7.82 \pm 0.45^{b}$ & $\begin{array}{l}85 \pm \\
0.70^{c}\end{array}$ & $\begin{array}{l}1.10 \pm \\
0.00^{\mathrm{b}}\end{array}$ \\
\hline T3 & $41.46 \pm 1.05^{a}$ & $2.41 \pm 0.06^{\mathrm{e}}$ & $0.70 \pm 0.00^{c}$ & $8.36 \pm 0.29^{c}$ & $\begin{array}{l}95 \pm \\
0.70^{b}\end{array}$ & $\begin{array}{l}1.10 \pm \\
0.00^{\mathrm{b}}\end{array}$ \\
\hline T4 & $62.34 \pm 2.37^{c}$ & $1.60 \pm 0.06^{b}$ & $0.59 \pm 0.07^{a}$ & $7.97 \pm 0.44^{b}$ & $100 \pm 00^{\mathrm{a}}$ & $\begin{array}{l}1.05 \pm \\
0.07^{b}\end{array}$ \\
\hline T5 & $43.48 \pm 1.45^{a}$ & $2.30 \pm 0.07^{e}$ & $0.61 \pm 0.05^{b}$ & $8.64 \pm 0.27^{c}$ & $100 \pm 00^{a}$ & $\begin{array}{l}0.90 \pm \\
0.00^{\mathrm{a}}\end{array}$ \\
\hline T6 & $\begin{array}{l}54.94 \pm \\
2.99^{\mathrm{b}}\end{array}$ & $1.82 \pm 0.09^{c}$ & $0.72 \pm 0.02^{c}$ & $7.45 \pm 0.02^{\mathrm{a}}$ & $100 \pm 00^{a}$ & $\begin{array}{l}1.00 \pm \\
0.14^{\mathrm{b}}\end{array}$ \\
\hline T7 & $\begin{array}{l}75.17 \pm \\
2.06^{d}\end{array}$ & $1.33 \pm 0.03^{a}$ & $0.67 \pm 0.03^{b}$ & $7.29 \pm 1.32^{\mathrm{a}}$ & $\begin{array}{l}95 \pm \\
0.70^{b}\end{array}$ & $\begin{array}{l}1.20 \pm \\
0.00^{\mathrm{b}}\end{array}$ \\
\hline T8 & $\begin{array}{l}79.38 \pm \\
1.46^{\mathrm{d}}\end{array}$ & $1.25 \pm 0.02^{\mathrm{a}}$ & $0.66 \pm 0.04^{b}$ & $8.23 \pm 0.07^{c}$ & $\begin{array}{l}95 \pm \\
0.70^{b}\end{array}$ & $\begin{array}{l}1.10 \pm \\
0.00^{\mathrm{b}}\end{array}$ \\
\hline T9 & $59.08 \pm 3.57^{b}$ & $1.69 \pm 0.10^{b}$ & $0.64 \pm 0.07^{b}$ & $7.05 \pm 0.14^{a}$ & $100 \pm 00^{a}$ & $\begin{array}{l}1.00 \pm \\
0.14^{b}\end{array}$ \\
\hline Control & $52.54 \pm 2.12^{b}$ & $1.91 \pm 0.08^{d}$ & $0.54 \pm 0.07^{a}$ & $7.15 \pm 0.25^{a}$ & $\begin{array}{l}75 \pm \\
0.70^{d}\end{array}$ & $\begin{array}{l}1.15 \pm \\
0.07^{b}\end{array}$ \\
\hline
\end{tabular}

Data expressed as Mean \pm Standard deviation.

Values with different superscripts within column differ significantly $(p<0.05)$

\subsection{Haematological parameters}

Among the haematological indices, highest number of RBC $\left(1.46 \pm 0.6 \times 10^{6} / \mathrm{mm}^{3}\right)$ was observed in T9. WBC were recorded the highest $\left(27.70 \pm 4.7 \times 10^{3} / \mathrm{mm}^{3}\right)$ in T8. Highest Haemoglobin $(11.90 \pm 1.9 \mathrm{~g} / \mathrm{dL})$ and haematocrit (41.27 $\pm 7.1 \%$ ) values showed maximum values in $\mathrm{T} 8$ respectively. $\mathrm{MCV}, \mathrm{MCH}$ and $\mathrm{MCHC}$ values were recorded highest for T2 $(317.06 \pm 32.43 \mathrm{fL})$, T8 $(85.98 \pm 3.30 \mathrm{pg})$ and T8 $(28.90 \pm 0.9 \mathrm{~g} / \mathrm{dL})$ respectively. Highest number of platelets $\left(273.96 \pm 21.22 \times 10^{3} / \mathrm{mm}^{3}\right)$ was recorded in T3. Blood protein $(6.42 \pm 0.1 \mathrm{~g} / \mathrm{dL})$ and Glucose $(118.47 \pm 12.09 \mathrm{mg} / \mathrm{dL})$ was recorded the highest in T7and control respectively. Data recorded was found to be statistically significant $(p<0.05)$.

Table 7. Haematological Indices recorded in GIFT Tilapia fed with experiment diets - I 


\begin{tabular}{llllll} 
Treatment & RBC & WBC & Platelets & Hb & Hct \\
\hline T1 & $1.31 \pm 0.2^{\mathrm{b}}$ & $26.39 \pm 2.4^{\mathrm{d}}$ & $268 \pm 34.21^{\mathrm{d}}$ & $8.33 \pm 1.2^{\mathrm{b}}$ & $36.53 \pm 7.9^{\mathrm{c}}$ \\
\hline T2 & $1.22 \pm 0.4^{\mathrm{a}}$ & $25.31 \pm 6.1^{\mathrm{c}}$ & $268 \pm 21.56^{\mathrm{d}}$ & $9.39 \pm 2.1^{\mathrm{c}}$ & $38.93 \pm 5.4^{\mathrm{d}}$ \\
\hline T3 & $1.21 \pm 0.7^{\mathrm{a}}$ & $25.17 \pm 4.2^{\mathrm{c}}$ & $273 \pm 21.22^{\mathrm{e}}$ & $10.16 \pm 2.2^{\mathrm{d}}$ & $38.33 \pm 7.7^{\mathrm{d}}$ \\
\hline T4 & $1.38 \pm 0.1^{\mathrm{b}}$ & $23.44 \pm 3.5^{\mathrm{b}}$ & $259 \pm 35.67^{\mathrm{c}}$ & $9.23 \pm 2.2^{\mathrm{c}}$ & $34.92 \pm 5.0^{\mathrm{b}}$ \\
\hline T5 & $1.39 \pm 0.9^{\mathrm{b}}$ & $21.11 \pm 3.7^{\mathrm{a}}$ & $269 \pm 12.34^{\mathrm{d}}$ & $8.40 \pm 3.4^{\mathrm{b}}$ & $35.70 \pm 7.7^{\mathrm{b}}$ \\
\hline T6 & $1.42 \pm 0.5^{\mathrm{c}}$ & $21.35 \pm 7.4^{\mathrm{a}}$ & $268 \pm 17.76^{\mathrm{d}}$ & $8.92 \pm 3.1^{\mathrm{b}}$ & $36.33 \pm 2.5^{\mathrm{c}}$ \\
\hline T7 & $1.36 \pm 0.4^{\mathrm{b}}$ & $27.38 \pm 1.5^{\mathrm{e}}$ & $233 \pm 34.09^{\mathrm{a}}$ & $7.90 \pm 3.0^{\mathrm{a}}$ & $36.39 \pm 3.6^{\mathrm{c}}$ \\
\hline T8 & $1.33 \pm 0.2^{\mathrm{b}}$ & $27.70 \pm 4.7^{\mathrm{e}}$ & $242 \pm 21.00^{\mathrm{b}}$ & $11.90 \pm 1.9^{\mathrm{e}}$ & $41.27 \pm 7.1^{\mathrm{e}}$ \\
\hline T9 & $1.46 \pm 0.6^{\mathrm{c}}$ & $27.60 \pm 4.2^{\mathrm{e}}$ & $233 \pm 22.67^{\mathrm{a}}$ & $8.20 \pm 2.7^{\mathrm{b}}$ & $38.67 \pm 9.0^{\mathrm{d}}$ \\
\hline Control & $1.34 \pm 0.9^{\mathrm{b}}$ & $26.48 \pm 2.1^{\mathrm{d}}$ & $252 \pm 19.06^{\mathrm{c}}$ & $7.80 \pm 1.1^{\mathrm{a}}$ & $32.33 \pm 3.7^{\mathrm{a}}$
\end{tabular}

Data expressed as Mean \pm Standard deviation.

Values with different superscripts within column differ significantly $(p<0.05)$

Table 8. Haematological Indices recorded in GIFT Tilapia fed with experiment diets - II

\begin{tabular}{llllll} 
Treatment & MCV & MCH & MCHC & Protein & Glucose \\
\hline T1 & $285.18 \pm 23.21^{\mathrm{d}}$ & $64.87 \pm 1.2^{\mathrm{b}}$ & $22.77 \pm 0.9^{\mathrm{b}}$ & $4.73 \pm 0.1^{\mathrm{a}}$ & $110.53 \pm 21.21^{\mathrm{c}}$ \\
\hline T2 & $317.06 \pm 32.43^{\mathrm{f}}$ & $76.41 \pm 3.3^{\mathrm{c}}$ & $24.09 \pm 1.7^{\mathrm{d}}$ & $4.73 \pm 0.2^{\mathrm{a}}$ & $105.43 \pm 23.28^{\mathrm{b}}$ \\
\hline T3 & $307.56 \pm 45.21^{\mathrm{f}}$ & $81.56 \pm 2.6^{\mathrm{d}}$ & $26.48 \pm 1.4^{\mathrm{e}}$ & $4.76 \pm 0.3^{\mathrm{a}}$ & $101.33 \pm 17.67^{\mathrm{b}}$ \\
\hline T4 & $258.47 \pm 24.32^{\mathrm{b}}$ & $68.18 \pm 2.4^{\mathrm{b}}$ & $26.40 \pm 0.7^{\mathrm{e}}$ & $6.03 \pm 0.3^{\mathrm{c}}$ & $98.33 \pm 13.78^{\mathrm{a}}$ \\
\hline T5 & $256.83 \pm 16.32^{\mathrm{b}}$ & $60.43 \pm 3.9^{\mathrm{b}}$ & $23.53 \pm 0.2^{\mathrm{c}}$ & $5.90 \pm 0.2^{\mathrm{b}}$ & $106.24 \pm 16.99^{\mathrm{b}}$ \\
\hline T6 & $260.59 \pm 21.00^{\mathrm{c}}$ & $64.05 \pm 5.0^{\mathrm{b}}$ & $24.41 \pm 1.8^{\mathrm{d}}$ & $6.32 \pm 0.2^{\mathrm{c}}$ & $101.14 \pm 25.00^{\mathrm{b}}$ \\
\hline T7 & $269.55 \pm 35.21^{\mathrm{c}}$ & $58.51 \pm 2.9^{\mathrm{a}}$ & $21.70 \pm 0.1^{\mathrm{a}}$ & $6.42 \pm 0.1^{\mathrm{c}}$ & $99.98 \pm 22.06^{\mathrm{a}}$ \\
\hline T8 & $296.99 \pm 33.33^{\mathrm{e}}$ & $85.98 \pm 3.3^{\mathrm{d}}$ & $28.90 \pm 0.9^{\mathrm{f}}$ & $4.87 \pm 0.2^{\mathrm{a}}$ & $96.28 \pm 21.09^{\mathrm{a}}$ \\
\hline T9 & $283.18 \pm 16.67^{\mathrm{d}}$ & $59.66 \pm 2.2^{\mathrm{a}}$ & $21.14 \pm 1.3^{\mathrm{a}}$ & $4.80 \pm 0.2^{\mathrm{a}}$ & $92.47 \pm 22.00^{\mathrm{a}}$ \\
\hline Control & $241.27 \pm 23.33^{\mathrm{a}}$ & $58.21 \pm 1.9^{\mathrm{a}}$ & $24.13 \pm 0.5^{\mathrm{d}}$ & $4.80 \pm 0.1^{\mathrm{a}}$ & $118.47 \pm 12.09^{\mathrm{c}}$
\end{tabular}

Data expressed as Mean \pm Standard deviation. 


\subsection{Digestive enzyme activity}

Digestive enzyme activity was significantly elevated in treatment groups. Highest Trypsin activity (4.414 \pm $0.5 \mathrm{U} / \mathrm{mg}$ protein) in the gut was observed in T2. Lipase activity was the highest $(9.414 \pm 1.1 \mathrm{U} / \mathrm{mg}$ protein) in T1. Highest activity of Amylase $(0.932 \pm 0.09 \mathrm{U} / \mathrm{mg}$ protein) was recorded in T6. Data recorded was found to be statistically significant $(p<0.05)$.

Table 9. Digestive Enzyme Activity

\begin{tabular}{llll} 
Treatment & \multicolumn{3}{l}{ Digestive Enzymes (U/mg protein) } \\
& Trypsin & Lipase & Amylase \\
\hline T1 & $2.334 \pm 0.5^{\mathrm{c}}$ & $9.414 \pm 1.1^{\mathrm{g}}$ & $0.124 \pm 0.01^{\mathrm{a}}$ \\
\hline T2 & $4.414 \pm 0.5^{\mathrm{d}}$ & $8.721 \pm 0.9^{\mathrm{f}}$ & $0.365 \pm 0.06^{\mathrm{b}}$ \\
\hline T3 & $2.067 \pm 0.9^{\mathrm{c}}$ & $9.213 \pm 0.6^{\mathrm{g}}$ & $0.357 \pm 0.03^{\mathrm{b}}$ \\
\hline T4 & $0.096 \pm 0.1^{\mathrm{a}}$ & $3.006 \pm 0.9^{\mathrm{b}}$ & $0.924 \pm 0.02^{\mathrm{e}}$ \\
\hline T5 & $0.072 \pm 0.2^{\mathrm{a}}$ & $3.333 \pm 0.7^{\mathrm{b}}$ & $0.896 \pm 0.11^{\mathrm{e}}$ \\
\hline T6 & $0.038 \pm 0.4^{\mathrm{a}}$ & $4.180 \pm 1.1^{\mathrm{c}}$ & $0.932 \pm 0.09^{\mathrm{e}}$ \\
\hline T7 & $1.639 \pm 0.4^{\mathrm{b}}$ & $6.454 \pm 0.9^{\mathrm{d}}$ & $0.433 \pm 0.05^{\mathrm{c}}$ \\
\hline T8 & $2.042 \pm 0.3^{\mathrm{c}}$ & $7.233 \pm 0.9^{\mathrm{e}}$ & $0.735 \pm 0.16^{\mathrm{d}}$ \\
\hline T9 & $1.131 \pm 0.1^{\mathrm{b}}$ & $7.254 \pm 1.2^{\mathrm{e}}$ & $0.774 \pm 0.90^{\mathrm{d}}$ \\
\hline Control & $0.750 \pm 0.1^{\mathrm{a}}$ & $2.465 \pm 0.1^{\mathrm{a}}$ & $0.224 \pm 0.80^{\mathrm{a}}$
\end{tabular}

Data expressed as Mean \pm Standard deviation.

Values with different superscripts within column differ significantly $(p<0.05)$

\subsection{Stress parameters}

For stress estimation, oxidative enzymes activity in gill, muscle and liver was recorded in different treatment groups and control. Maximum values were recorded in liver followed by gill and muscle. Highest Catalase activity in the liver $(2.6630 \pm 0.64 \mathrm{U} / \mathrm{mg}$ protein), gill $(2.4187 \pm 0.025 \mathrm{U} / \mathrm{mg}$ protein) and muscle $(1.9150 \pm 0.013 \mathrm{U} / \mathrm{mg}$ protein) was recorded in T9. Highest LDH activity in the liver $(16.8560 \pm 0.192 \mathrm{U} / \mathrm{mg}$ protein), gill (14.039 $\pm 0.061 \mathrm{U} / \mathrm{mg}$ protein) and muscle $(13.7903 \pm 0.020 \mathrm{U} / \mathrm{mg}$ protein) was recorded in 
T9. $\mathrm{MDH}$ activity was also recorded the highest in T9 in liver $(2.9407 \pm 0.068 \mathrm{U} / \mathrm{mg}$ protein) and gill (2.4620 $\pm 0.077 \mathrm{U} / \mathrm{mg}$ protein) In muscle, highest MDH activity was found in T8 $(1.9113 \pm 0.140 \mathrm{U} / \mathrm{mg}$ protein). Highest SOD activity in the liver ( $2.9820 \pm 0.068 \mathrm{U} / \mathrm{mg}$ protein), gill $(2.2610 \pm 0.032 \mathrm{U} / \mathrm{mg}$ protein) and muscle $(2.0767 \pm 0.068 \mathrm{U} / \mathrm{mg}$ protein) was recorded in T9. NBT activity also showed the maximum values in T9 in liver ( $1.412 \pm 0.126 \mathrm{U} / \mathrm{mg}$ protein), gill $(1.116 \pm 0.159 \mathrm{U} / \mathrm{mg}$ protein) and muscle $(0.879 \pm$ $0.018 \mathrm{U} / \mathrm{mg}$ protein). Data recorded was found to be statistically significant $(p<0.05)$.

\section{Table 10. Catalase Activity}

Treatment Catalase Activity (U/mg protein)

\begin{tabular}{llll}
\hline & Liver & Gill & Muscle \\
\hline T1 & $1.2727 \pm 0.026^{\mathrm{b}}$ & $0.3753 \pm 0.034^{\mathrm{a}}$ & $0.3497 \pm 0.064^{\mathrm{a}}$ \\
\hline T2 & $1.3140 \pm 0.011^{\mathrm{b}}$ & $0.9617 \pm 0.012^{\mathrm{a}}$ & $0.6597 \pm 0.056^{\mathrm{b}}$ \\
\hline T3 & $1.5463 \pm 0.065^{\mathrm{b}}$ & $1.2173 \pm 0.019^{\mathrm{b}}$ & $0.7960 \pm 0.006^{\mathrm{b}}$ \\
\hline T4 & $2.0073 \pm 0.003^{\mathrm{c}}$ & $0.6547 \pm 0.476^{\mathrm{a}}$ & $0.7770 \pm 0.009^{\mathrm{b}}$ \\
\hline T5 & $2.3177 \pm 0.091^{\mathrm{c}}$ & $1.6087 \pm 0.560^{\mathrm{b}}$ & $1.0473 \pm 0.013^{\mathrm{c}}$ \\
\hline T6 & $2.5423 \pm 0.071^{\mathrm{c}}$ & $2.3133 \pm 0.077^{\mathrm{c}}$ & $1.5087 \pm 0.008^{\mathrm{c}}$ \\
\hline T7 & $2.5043 \pm 0.115^{\mathrm{c}}$ & $1.3917 \pm 0.020^{\mathrm{b}}$ & $0.9930 \pm 0.006^{\mathrm{b}}$ \\
\hline T8 & $2.3147 \pm 0.019^{\mathrm{c}}$ & $1.9623 \pm 0.055^{\mathrm{b}}$ & $1.5083 \pm 0.017^{\mathrm{c}}$ \\
\hline T9 & $2.6630 \pm 0.064^{\mathrm{c}}$ & $2.4187 \pm 0.025^{\mathrm{c}}$ & $1.9150 \pm 0.013^{\mathrm{c}}$ \\
\hline Control & $0.5593 \pm 0.042^{\mathrm{a}}$ & $0.4360 \pm 0.044^{\mathrm{a}}$ & $0.2390 \pm 0.006^{\mathrm{a}}$
\end{tabular}

Data expressed as Mean \pm Standard deviation.

Values with different superscripts within column differ significantly $(p<0.05)$

Table 11. LDH Activity 


\section{Treatment LDH Activity (U/mg protein)}

\begin{tabular}{llll}
\hline & Liver & Gill & Muscle \\
\hline T1 & $8.3357 \pm 0.037^{\mathrm{c}}$ & $6.8157 \pm 0.248^{\mathrm{b}}$ & $6.6443 \pm 0.037^{\mathrm{b}}$ \\
\hline T2 & $11.4577 \pm 0.189^{\mathrm{c}}$ & $8.7290 \pm 0.189^{\mathrm{c}}$ & $6.4783 \pm 0.040^{\mathrm{b}}$ \\
\hline T3 & $12.1683 \pm 0.133^{\mathrm{c}}$ & $9.4593 \pm 0.237^{\mathrm{c}}$ & $6.5003 \pm 0.109^{\mathrm{b}}$ \\
\hline T4 & $4.1970 \pm 0.155^{\mathrm{a}}$ & $3.8277 \pm 0.097^{\mathrm{a}}$ & $3.2967 \pm 0.046^{\mathrm{a}}$ \\
\hline T5 & $5.8340 \pm 0.077^{\mathrm{b}}$ & $3.9843 \pm 0.051^{\mathrm{a}}$ & $3.6443 \pm 0.008^{\mathrm{a}}$ \\
\hline T6 & $5.8350 \pm 0.027^{\mathrm{b}}$ & $5.3530 \pm 0.053^{\mathrm{b}}$ & $3.1123 \pm 0.048^{\mathrm{a}}$ \\
\hline T7 & $14.2883 \pm 0.269^{\mathrm{d}}$ & $12.235 \pm 0.222^{\mathrm{e}}$ & $9.7720 \pm 0.113^{\mathrm{c}}$ \\
\hline T8 & $14.1853 \pm 0.130^{\mathrm{d}}$ & $10.991 \pm 0.019^{\mathrm{d}}$ & $11.0640 \pm 0.094^{\mathrm{d}}$ \\
\hline T9 & $16.8560 \pm 0.192^{\mathrm{e}}$ & $14.039 \pm 0.061^{\mathrm{f}}$ & $13.7903 \pm 0.020^{\mathrm{e}}$ \\
\hline Control & $4.2100 \pm 0.0781^{\mathrm{a}}$ & $3.572 \pm 0.299^{\mathrm{a}}$ & $3.3820 \pm 0.208^{\mathrm{a}}$
\end{tabular}

Data expressed as Mean \pm Standard deviation.

Values with different superscripts within column differ significantly $(p<0.05)$

Table 12. MDH Activity 
Treatment MDH Activity (U/mg protein)

\begin{tabular}{llll}
\hline & Liver & Gill & Muscle \\
\hline T1 & $1.0487 \pm 0.046^{\mathrm{b}}$ & $0.6760 \pm 0.107^{\mathrm{a}}$ & $0.2263 \pm 0.002^{\mathrm{a}}$ \\
\hline T2 & $1.0220 \pm 0.054^{\mathrm{b}}$ & $0.6530 \pm 0.051^{\mathrm{a}}$ & $0.4280 \pm 0.031^{\mathrm{a}}$ \\
\hline T3 & $1.0653 \pm 0.015^{\mathrm{b}}$ & $0.9887 \pm 0.074^{\mathrm{a}}$ & $0.6290 \pm 0.027^{\mathrm{a}}$ \\
\hline T4 & $1.0083 \pm 0.005^{\mathrm{b}}$ & $0.9763 \pm 0.033^{\mathrm{a}}$ & $0.6217 \pm 0.022^{\mathrm{a}}$ \\
\hline T5 & $1.1117 \pm 0.052^{\mathrm{b}}$ & $1.0797 \pm 0.052^{\mathrm{b}}$ & $0.6543 \pm 0.057^{\mathrm{a}}$ \\
\hline T6 & $1.2993 \pm 0.029^{\mathrm{b}}$ & $1.0273 \pm 0.005^{\mathrm{b}}$ & $1.0483 \pm 0.100^{\mathrm{b}}$ \\
\hline T7 & $2.2333 \pm 0.100^{\mathrm{c}}$ & $1.9840 \pm 0.030^{\mathrm{b}}$ & $1.5887 \pm 0.029^{\mathrm{b}}$ \\
\hline T8 & $2.6523 \pm 0.276^{\mathrm{c}}$ & $2.1697 \pm 0.027^{\mathrm{c}}$ & $1.9113 \pm 0.140^{\mathrm{b}}$ \\
\hline T9 & $2.9407 \pm 0.068^{\mathrm{c}}$ & $2.4620 \pm 0.077^{\mathrm{c}}$ & $1.6053 \pm 0.072^{\mathrm{b}}$ \\
\hline Control & $0.9000 \pm 0.030^{\mathrm{a}}$ & $0.9740 \pm 0.054^{\mathrm{a}}$ & $0.5077 \pm 0.024^{\mathrm{a}}$
\end{tabular}

Data expressed as Mean \pm Standard deviation.

Values with different superscripts within column differ significantly $(p<0.05)$

Table 13. SOD Activity 


\section{Treatment SOD Activity (U/mg protein)}

\begin{tabular}{llll} 
& Liver & Gill & Muscle \\
\hline T1 & $0.9870 \pm 0.035^{\mathrm{a}}$ & $0.7553 \pm 0.051^{\mathrm{a}}$ & $0.2637 \pm 0.092^{\mathrm{a}}$ \\
\hline T2 & $1.8740 \pm 0.056^{\mathrm{b}}$ & $1.1847 \pm 0.134^{\mathrm{b}}$ & $0.5943 \pm 0.014^{\mathrm{a}}$ \\
\hline T3 & $1.9690 \pm 0.066^{\mathrm{b}}$ & $1.6747 \pm 0.093^{\mathrm{b}}$ & $0.6970 \pm 0.001^{\mathrm{a}}$ \\
\hline T4 & $1.4023 \pm 0.112^{\mathrm{b}}$ & $1.0480 \pm 0.019^{\mathrm{b}}$ & $0.8903 \pm 0.022^{\mathrm{a}}$ \\
\hline T5 & $1.8740 \pm 0.031^{\mathrm{b}}$ & $1.4243 \pm 0.025^{\mathrm{b}}$ & $0.9337 \pm 0.007^{\mathrm{a}}$ \\
\hline T6 & $1.9693 \pm 0.072^{\mathrm{b}}$ & $1.6423 \pm 0.061^{\mathrm{b}}$ & $0.9913 \pm 0.010^{\mathrm{a}}$ \\
\hline T7 & $2.0540 \pm 0.011^{\mathrm{c}}$ & $1.7460 \pm 0.067^{\mathrm{b}}$ & $1.4553 \pm 0.051^{\mathrm{b}}$ \\
\hline T8 & $2.4397 \pm 0.089^{\mathrm{c}}$ & $1.9833 \pm 0.016^{\mathrm{b}}$ & $1.8540 \pm 0.072^{\mathrm{b}}$ \\
\hline T9 & $2.9820 \pm 0.068^{\mathrm{c}}$ & $2.2610 \pm 0.032^{\mathrm{c}}$ & $2.0767 \pm 0.068^{\mathrm{c}}$ \\
\hline Control & $0.9997 \pm 0.007^{\mathrm{a}}$ & $0.6413 \pm 0.051^{\mathrm{a}}$ & $0.4007 \pm 0.004^{\mathrm{a}}$
\end{tabular}

Data expressed as Mean \pm Standard deviation.

Values with different superscripts within column differ significantly $(p<0.05)$

Table 14. NBT Activity 


\begin{tabular}{llll} 
Treatment & \multicolumn{3}{l}{ NBT Activity (U/mg protein) } \\
& Liver & Gill & Muscle \\
\hline T1 & $1.027 \pm 0.002^{\mathrm{b}}$ & $0.776 \pm 0.026^{\mathrm{a}}$ & $0.442 \pm 0.014^{\mathrm{a}}$ \\
\hline T2 & $1.095 \pm 0.012^{\mathrm{b}}$ & $0.881 \pm 0.159^{\mathrm{a}}$ & $0.633 \pm 0.026^{\mathrm{b}}$ \\
\hline T3 & $1.194 \pm 0.235^{\mathrm{b}}$ & $0.899 \pm 0.231^{\mathrm{a}}$ & $0.694 \pm 0.125^{\mathrm{b}}$ \\
\hline T4 & $0.613 \pm 0.065^{\mathrm{a}}$ & $0.811 \pm 0.006^{\mathrm{a}}$ & $0.322 \pm 0.009^{\mathrm{a}}$ \\
\hline T5 & $0.681 \pm 0.010^{\mathrm{a}}$ & $0.887 \pm 0.004^{\mathrm{a}}$ & $0.382 \pm 0.006^{\mathrm{a}}$ \\
\hline T6 & $0.933 \pm 0.009^{\mathrm{a}}$ & $0.924 \pm 0.012^{\mathrm{a}}$ & $0.497 \pm 0.007^{\mathrm{a}}$ \\
\hline T7 & $1.397 \pm 0.005^{\mathrm{b}}$ & $1.086 \pm 0.014^{\mathrm{b}}$ & $0.644 \pm 0.002^{\mathrm{b}}$ \\
\hline T8 & $1.406 \pm 0.004^{\mathrm{b}}$ & $1.103 \pm 0.026^{\mathrm{b}}$ & $0.687 \pm 0.001^{\mathrm{b}}$ \\
\hline T9 & $1.412 \pm 0.126^{\mathrm{b}}$ & $1.116 \pm 0.159^{\mathrm{b}}$ & $0.879 \pm 0.018^{\mathrm{b}}$ \\
\hline Control & $0.924 \pm 0.008^{\mathrm{a}}$ & $0.717 \pm 0.008^{\mathrm{a}}$ & $0.162 \pm 0.025^{\mathrm{a}}$
\end{tabular}

Data expressed as Mean \pm Standard deviation.

Values with different superscripts within column differ significantly $(p<0.05)$

\subsection{Visible difference in body coloration}

Treatment groups fed with different seed supplemented diets showed visible changes in body coloration as compared to control. The groups fed with Celery seed supplemented diets showed a darker colour with prominently darker bands. Fenugreek seed supplemented diet group gradually switched the body colouration to orange-brown however no change in bands was noticed. Coriander seed fed group showed shiny/metallic body coloration with almost no bands visible.

\section{Discussion}

The results obtained in the present study clearly indicate that the use of organic herbal spice seeds in fish diets have pronounced effect on the growth, haematological performance, digestive enzyme activity and stress parameters of the fish. In the present study, T8 (Coriander seed powder, $2 \%$ ) showed the best growth performance throughout the experiment. At the end of the experiment, maximum length and weight was observed in T8 was $10.04 \pm 0.34 \mathrm{~cm}$ and $11.48 \pm 0.19 \mathrm{~g}$ respectively which was significantly higher than control $(7.46 \pm 0.42 \mathrm{~cm}$ and $8.53 \pm 0.27 \mathrm{~g}$ respectively). The results are in agreement with Al-Shakarchi and Mohammed (2021) who has fed coriander supplemented diet (1.75\%) to Common carp (Cyprinus carpio) which resulted in enhanced growth performance as compared to the control group. Similar results have 
been reported by Raissy et al. (2021) who fed Common carp (Cyprinus carpio) with Coriander (Coriandrum sativum), Oak acorn (Quercus brantii) and Mallow (Malva sy/vestris). T8 also showed the best results in terms of bio-growth parameters such as Weight Gain (8.103 $\pm 0.160 \mathrm{~g})$, Daily Weight Gain $(4.00 \pm 0.04 \%)$, Specific Growth Rate (2.04 $\pm 0.01 \%)$, Relative Growth Rate (L) (191.75 $\pm 5.86 \%)$, Relative Growth Rate (W) $(240.34 \pm 2.59 \%)$ etc. These results are in accordance with Farsani et al., (2019) who fed Rainbow trout (Onchorhynchus mykiss) with different levels of coriander supplemented diets and found out significantly higher values of Total weight gain, Daily weight gain and Specific Growth Rate (SGR) in the treatment group fed with $2 \%$ coriander seed incorporated diets. Enhanced growth performance in fish fed with coriander can be attributed to the effect of bactericidal property of coriander seeds on the intestinal flora which leads to better nutrient absorption and in turn higher metabolism and better feed utility responsible for enhanced growth performance (Citarasu T, 2010; Awad and Awaad, 2017; Begnami et al., 2018). Better growth prospects in fish fed with coriander seeds could be related to the presence of some specific biochemical compounds such as Terpenes and Coriandrons present in coriander seeds (Nadeem et al., 2013). It is evident that the spice or herbal seeds have appetite-inducing and digestion-stimulating properties that play an important role in feed utilization and conversion hence leading to better growth performance (Jain et al., 2008).

Haematological indices are a significant tool for monitoring fish health in general and response to nutrition in particular (Secombes 1996; Cuesta 2005; Lin et al., 2015). As per the results obtained from the present study, highest RBC count $\left(1.46 \pm 0.6 \times 10^{6} / \mathrm{mm}^{3}\right)$ was recorded in T9 (Coriander seed powder, $2.5 \%$ ) while as highest WBC count $\left(27.70 \pm 4.7 \times 10^{3} / \mathrm{mm}^{3}\right)$ was observed in T8 (Coriander seed powder, $2.0 \%$ ). These findings are in agreement with Farsani et al. (2019) who have fed Rainbow trout (Onchorhynchus mykiss) with different levels of coriander supplemented diets and found out the best haematological performance in diets supplemented with $2 \%$ coriander. T8 also showed the highest values of haemoglobin and haematocrit and followed an increasing trend throughout the experiment. Increase in blood cell counts can be attributed to the effect of bio-active compounds in coriander seed on the lymphoid organs of the fish (Ellis, 2001) which results in increased production of cells in the blood. RBC count not only indicates the health status of the fish but is a reliable indicator of stress in fish (Talpur and Ikhwanuddin, 2013). Any abnormality in RBC count in blood either indicates anaemia in fish or presence of stress (Talpur and Ikwanuddin, 2013). In the present study, the RBC counts of the fish fed with different treatment diets did not vary greatly from each other in terms of blood cell counts, only a slight variation among different treatment groups and between treatment groups and control was observed (Maximum value, T9 $\left(1.46 \pm 0.6 \times 10^{6} / \mathrm{mm}^{3}\right)$, Minimum value, T3 $\left(1.21 \pm 0.7 \times 10^{6} / \mathrm{mm}^{3}\right)$. Hence, it can be assured that as per the results obtained for blood indices, the fish in the present study did not undergo any stress. Similar results have been put forth by Raissy et al. (2021) who related the highest growth performance in coriander supplemented diet group to the effects of coriander essential oils (in coriander seeds) that have a stimulatory effect on the haematopoietic organs of the fish responsible for the production of RBCs and increase the oxygen carrying capacity of the cells. Total Protein, Albumin, and Globulin are considered the most important tools of monitoring the fish health prospect in terms of immunity (Ahmadi et al., 2012; Rao et al., 2006; Sahu et al., 2007 ). In the present study, Blood protein (Albumin and Globulin) was observed 
the highest $(6.42 \pm 0.1 \mathrm{~g} / \mathrm{dL})$ in T7 (Coriander seed powder, $1.5 \%)$ in comparison to other treatment groups and control. Highest value of glucose $(118.47 \pm 12.09 \mathrm{mg} / \mathrm{dl})$ was recorded in control throughout the experiment. Lowest glucose level $(92.47 \pm 22.00 \mathrm{mg} / \mathrm{dl})$ was recorded in T9 (Coriander seed powder, $2.5 \%$ ). Significant decrease in blood glucose levels in treatment group fed with $2.5 \%$ coriander seed powder supplementation may be due to the hypoglycemic property of coriander seeds (Laribi et al., 2015). Specifically, the hypoglycemic effect of coriander seeds on fish blood glucose can be better explained as a result of the combined effect of certain bioactive compounds present in coriander seeds such as Linalool, Geranyl Acetate and y-terpinene (Gallagher et al., 2003; Pandeya, 2013).In certain fishes including Tilapia, wide distribution of different digestive enzymes like protease, trypsin, lipase and amylase in addition to intestinal length promotes the possibility of digesting a wide range of food sources and significantly improves the metabolic process of food breakdown into blood streams responsible for tissue formation and overall growth (Montoya-Mejia et al., 2017).

In fishes, the digestion of nutrients begins with the activity of digestive enzymes including Trypsin, Chymotrypsin, Amylase and Lipase secreted by the pancreas into the stomach and continues till the intestines (Moriarty, 1973; Nagase, 1964). In the present study, different treatment groups fed with different herb supplemented diets were subjected to digestive enzyme analysis (Trypsin, Lipase and Amylase). Trypsin activity is considered as one of the conditional indicators of fish, and its secretion is directly proportional to the activity of the pancreas (Sunde et al., 2001). In the present study, highest Trypsin activity (4.414 $\pm 0.5 \mathrm{U} / \mathrm{mg}$ protein) in the gut was observed in T2 (Celery seed powder, $2 \%$ ) and lowest $(0.038 \pm 0.4 \mathrm{U} / \mathrm{mg}$ protein) in T6 (Fenugreek seed powder, $2.5 \%)$. Enhanced Trypsin activity in Celery fed group is in line with the study carried out by Mohamed et al., (2018) who fed Common Carp (Cyprinus carpio) with celery supplemented diet in three different proportions and observed a consistent increase in the Trypsin levels with increase in Celery supplementation in diets. Trypsin levels in treatment fish fed with Fenugreek supplemented diets were observed the lowest compared to other treatment groups and control diet fed groups. Lowest trypsin levels may be due to the presence of characteristic Trypsin inhibitors present in Fenugreek seed (Al-Maiman, 2004; Mansour and El-Adawy, 1994; Oddepally et al., 2013; Pallavi and Rajender, 2021). Lipase enzyme is mainly secreted by the pancreas and plays a major role in lipid breakdown, especially tri-acyl-glycerols, necessary for better digestion (Awad, 2010). In the present research study, highest Lipase activity $(0.932 \pm 0.09 \mathrm{U} / \mathrm{mg}$ protein) was recorded in T6 (Fenugreek seed powder, $2.5 \%)$ while as the lowest Lipase activity $(2.456 \pm 0.1 \mathrm{U} / \mathrm{mg}$ protein) was found in control group. The result can be related to the possible explanation that among all the three herb ingredients used in the present study, Fenugreek constitutes the highest (6-7\%) lipid content (USDA, 2012) for the breakdown of which, Lipase production in fish digestive tract shows a significant increase with the increase in supplementation levels (Mehboob et al., 2017) compared to other experiment ingredients (Celery and Coriander seed) with negligible lipid content. Similar results have been reported by Al-Saraji and Nasir (2013) who fed the Common Carp (Cyprinus carpio) with different protein source supplemented diets and observed an increase in Lipase levels in Fenugreek supplemented diet fed group compared to other treatment groups and control. Amylase enzyme production in fish is stimulated by the presence of glycogen, glycolytic chains, starch and higher dietary protein content in fish diet (Krogdahl et al., 2005). Maximum Amylase enzyme activity $(0.932 \pm 0.09 \mathrm{U} / \mathrm{mg}$ protein) was observed in T6 (Fenugreek seed 
powder, $2.5 \%)$ and minimum $(0.124 \pm 0.01 \mathrm{U} / \mathrm{mg}$ protein) in $\mathrm{T} 1$ (Celery seed powder, $1.5 \%)$. The results are evident by the fact that Fenugreek seeds are exceptionally high (23-26\%) in protein content (USDA, 2012) in comparison to Celery and Coriander with meager dietary protein content. The results obtained are in line with the results reported by Kawai (1973) who found an increase in Amylase enzyme in the gut of Rainbow trout ( $O$. mykiss) fed with Fenugreek as a source of dietary plant protein.

Oxidative stress is accompanied by an increase in the production of Reactive Oxygen Species (ROS). In the present study, highest values for oxidative enzymes were recorded in liver followed by gill and muscle. In the liver, highest Catalase activity was recorded in T9 (2.6630 $\pm 0.64 \mathrm{U} / \mathrm{mg}$ protein). In gill, highest activity was recorded in T9 $(2.4187 \pm 0.025 \mathrm{U} / \mathrm{mg}$ protein) and in muscle, highest Catalase activity was found in T9 (1.9150 $\pm 0.013 \mathrm{U} / \mathrm{mg}$ protein). Although, very scanty reports are available on the relationship between oxidative enzymes and herbs used in the present study, however, highest value of Catalase in T9 (Coriander seed powder, 2.5\%) can be possibly related to the high content of catalase in celery (Kolarovic et al., 2009). Under stress, anaerobic glycolysis supplies energy accompanied by a significant accumulation of lactate (LDH) under hypoxic conditions (Wang et al., 2013). In the liver, highest LDH activity was recorded in T9 (16.8560 $\pm 0.192 \mathrm{U} / \mathrm{mg}$ protein). In gill, highest activity was recorded in T9 (14.039 $\pm 0.061 \mathrm{U} / \mathrm{mg}$ protein) and in muscle, highest LDH activity was found also found in T9 (13.7903 \pm $0.020 \mathrm{U} / \mathrm{mg}$ protein). Zhao et al. (2020) reported that fish exposed to hypoxic and ammonia conditions exhibits higher lactate concentration and LDH activity, confirming the occurrence of anaerobic glycolysis under simultaneous exposure. In the liver, highest MDH activity was recorded in T9 $(2.9407 \pm 0.068 \mathrm{U} / \mathrm{mg}$ protein) followed by gill $(2.4620 \pm 0.077 \mathrm{U} / \mathrm{mg}$ protein), where as in muscle, highest MDH activity was found in T8 ( $1.9113 \pm 0.140 \mathrm{U} / \mathrm{mg}$ protein). High stocking density can also cause oxidative stress in fish, because of the imbalance between reactive oxygen species and antioxidant defense systems (Mittler, 2002; Jia et al., 2016). In fish body these are removed by the antioxidant enzymes such as SOD, GPx, and Catalase (Birnie-Gauvin et al., 2017). In the liver, highest SOD activity was recorded in T9 $(2.9820 \pm 0.068$ $\mathrm{U} / \mathrm{mg}$ protein) followed by gill $(2.2610 \pm 0.032 \mathrm{U} / \mathrm{mg}$ protein) and muscle $(2.0767 \pm 0.068 \mathrm{U} / \mathrm{mg}$ protein). Wang et al. (2013) observed an increase in the activity of antioxidant enzymes in fish under high stocking density conditions, indicating that the adaptive response of the antioxidant system protects the body from oxygen free radicals during stress. In the liver, highest NBT activity was recorded in T9 $(1.412 \pm 0.126$ $\mathrm{U} / \mathrm{mg}$ protein) followed by gill $(1.116 \pm 0.159 \mathrm{U} / \mathrm{mg}$ protein) and muscle $(0.879 \pm 0.018 \mathrm{U} / \mathrm{mg}$ protein). Previous research studies have revealed that acute hypoxia induced oxidative stress has altered antioxidant enzyme activities in Largemouth Bass (Micropterus salmoides) (Yang et al., 2017). Similar results were also obtained for Spotted Croaker (Leiostomus xanthurus) exposed to hypoxia (Cooper et al., 2002).

\section{Declarations}

\section{CONFLICTS OF INTEREST}

The authors declare no conflict(s) of interest in the present study.

\section{AUTHOR DECLARATION}

Page 21/28 


\section{Funding:}

The current research work was financially supported (partially) by the Department of Aquaculture, Fisheries College and Research Institute, Thoothukudi, Tamil Nadu, India - 628008.

\section{Conflicts of interest/Competing interests:}

The authors (Corresponding and Co-authors) declare no conflicts of interest related to the present research study.

\section{Ethics approval:}

Not applicable

\section{Availability of data and material:}

The author declares that all the information produced in the current research paper is original. In any case where the data has been copied from already existing literary sources, the same text is followed by the source reference.

\section{Code availability:}

Not applicable

\section{Authors' contributions:}

All the research works mentioned in the current research paper have been carried out by the corresponding author. Literary and scientific corrections in paper writing have been carried out by the co-authors.

\section{CONSENT TO PARTICIPATE}

The author puts forth the consent to participate in a survey, research or related works anytime in future conditioned to availability.

\section{CONSENT FOR PUBLICATION}

The author puts forth the consent to publish the present work in the current journal.

\section{References}

1. Abarike, E.D., Jian, J., Tang, J., Cai, J., Yu, H., Lihua, C. and Jun, L., 2018. Influence of traditional Chinese medicine and Bacillus species (TCMBS) on growth, immune response and disease resistance in Nile tilapia, Oreochromis niloticus. Aquaculture Research, 49(7), pp.2366-2375.

2. Abdelwahab, A.M. and El-Bahr, S.M., 2012. Influence of black cumin seeds (Nigella sativa) and turmeric (Curcuma longa Linn.) mixture on performance and serum biochemistry of Asian sea bass, Lates calcarifer. World Journal of Fish and Marine Sciences, 4(5), pp.496-503.

3. Ahmadi, K., Banaee, M., Vosoghei, A.R., Mirvaghefei, A.R. and Ataeimehr, B., 2012. Evaluation of the immunomodulatory effects of silymarin extract (Silybum marianum) on some immune parameters of 
rainbow trout, Oncorhynchus mykiss (Actinopterygii: Salmoniformes: Salmonidae). Acta Ichthyologica Et Piscatoria, 42(2).

4. Ahmed, B., Alam, T., Varshney, M. and Khan, S.A., 2002. Hepatoprotective activity of two plants belonging to the Apiaceae and the Euphorbiaceae family. Journal of Ethnopharmacology, 79(3), pp.313-316.

5. Al-Maiman, S.A., 2004. Inactivation of trypsin and chymotrypsin inhibitors in fenugreek (Trigonella foenum graecum L.) defatted seed flour. Food Sci Agric Res Center, King Saud Univ Res Bult, 130, pp.5-17.

6. Al-Mofleh, I.A., Alhaider, A.A., Mossa, J.S., Al-Sohaibani, M.O., Rafatullah, S. and Qureshi, S., 2006. Protection of gastric mucosal damage by Coriandrum sativum L. pretreatment. Environmental Toxicology and Pharmacology, 22(1), pp.64-69.

7. Al-Saraji, A.Y.J. and Nasir, N.A.N., 2013. Effect of different dietary proteins and fats on the digestive enzymes activities in the common carp fingerlings (Cyprinus carpio L.) reared in floating cages. Mesopotamian Journal of Marine Science, 28(2), pp.121-130.

8. Al-Shakarchi, H.H. and Mohammad, M.A., 2021. Effects of different levels of Cinnamon and Coriander powders on growth performance, feed utilization, survival rate and body chemical composition of Common carp (Cyprinus carpio I.) Plant Archives, 21(1), pp.46-54.

9. Anderson and Siwicki, AK., 1995. Basic haematology and serology for fish health programs. In Shariff, M., Arthur, JR., Subasinghe, RP. (Eds.). Diseases in Asian aquaculture II. Fish Health Section. Manila: Asian Fisheries Society. p. 185-202.

10. AOAC 2016, Official methods of analysis, Association of official Analytical Chemists, 20th edition, Geitherburg, MD,USA.

11. APHA, 1998. Standard methods for the estimation of water and waste waters, 20th edn. American Public Health Association, New York. 157-169

12. Awad, E. and Austin, B., 2010. Use of lupin, Lupinus perennis, mango, Mangifera indica, and stinging nettle, Urtica dioica, as feed additives to prevent Aeromonas hydrophila infection in rainbow trout, Oncorhynchus mykiss (Walbaum). Journal of fish diseases, 33(5), pp.413-420.

13. Awad, E. and Awaad, A., 2017. Role of medicinal plants on growth performance and immune status in fish. Fish \& shellfish immunology, 67, pp.40-54.

14. Aydemir, T. and Becerik, S., 2011. Phenolic content and antioxidant activity of different extracts from Ocimum basilicum, Apium graveolens and Lepidium sativum seeds. Journal of food biochemistry, 35(1), pp.62-79.

15. Begnami, A.F., Spindola, H.M., Ruiz, A.L.T.G., de Carvalho, J.E., Groppo, F.C. and Rehder, V.L.G., 2018. Antinociceptive and anti-edema properties of the ethyl acetate fraction obtained from extracts of Coriandrum sativum Linn. Leaves. Biomedicine \& Pharmacotherapy, 103, pp.1617-1622.

16. Behera, B.K., Pradhan, P.K., Swaminathan, T.R., Sood, N., Paria, P., Das, A., Verma, D.K., Kumar, R., Yadav, M.K., Dev, A.K. and Parida, P.K., 2018. Emergence of tilapia lake virus associated with 
mortalities of farmed Nile tilapia Oreochromis niloticus (Linnaeus 1758) in India. Aquaculture, 484, pp.168-174.

17. Bernstein, L.H. and Grisham, M.B., 1978. Kinetic determination of malate dehydrogenase isozymes. Journal of molecular and cellular cardiology, 10(10), pp.931-944.

18. Birnie Gauvin, K., Costantini, D., Cooke, S.J. and Willmore, W.G., 2017. A comparative and evolutionary approach to oxidative stress in fish: a review. Fish and Fisheries, 18(5), pp. 928-942.

19. Blaxhall, P.C. and Daisley, K.W., 1973. Routine haematological methods for use with fish blood. Journal of fish biology, 5(6), pp.771-781.

20. Boyd, C.E., 1982. Water quality management for pond fish culture. Elsevier Scientific Publishing Co.

21. Chang, J., 2000. Medicinal herbs: drugs or dietary supplements? Biochemical pharmacology, 59(3), pp.211-219.

22. Citarasu, T., 2010. Herbal biomedicines: a new opportunity for aquaculture industry. Aquaculture International, 18(3), pp.403-414.

23. Cooper, R.U., Clough, L.M., Farwell, M.A., West, T.L., 2002. Hypoxia-induced metabolic and antioxidant enzymatic activities in the estuarine fish Leiostomus xanthurus. Journal of experimental marine biology and ecology, 279 (1-2), 1-20.

24. Cuesta, A., Rodríguez, A., Esteban, M.A. and Meseguer, J., 2005. In vivo effects of propolis, a honeybee product, on gilthead seabream innate immune responses. Fish \& Shellfish Immunology, 18(1), pp.7180.

25. Drabkin, D.L., 1946. Spectrophotometric studies XIV. The crystallographic and optical properties of the hemoglobin of man in comparison with those of other species. Journal of biological chemistry, 164(2), pp.703-723.

26. Ellis, A.E., 2001. Innate host defense mechanisms of fish against viruses and bacteria. Developmental \& Comparative Immunology, 25(8-9), pp.827-839.

27. El-Sayed AFM. Stress and diseases. In: El-Sayed AFM. editor, Tilapia Culture. Cambridge: CABI Publishing, 2006; p. 149-151.

28. El-Sayed, A.F.M., 2019. Tilapia culture. Book currently in Academic Press.

29. FAO, 2016. The State of World Fisheries and Aquaculture 2016. Contributing to food security and nutrition for all. Rome. 200 pp.

30. Farsani, M.N., Hoseinifar, S.H., Rashidian, G., Farsani, H.G., Ashouri, G. and Van Doan, H., 2019. Dietary effects of Coriandrum sativum extract on growth performance, physiological and innate immune responses and resistance of rainbow trout (Oncorhynchus mykiss) against Yersinia ruckeri. Fish \& shellfish immunology, 91, pp.233-240.

31. Folch, J., Lees, M. and Stanley, G.S., 1957. A simple method for the isolation and purification of total lipids from animal tissues. Journal of biological chemistry, 226(1), pp.497-509.

32. Gallagher, A.M., Flatt, P.R., Duffy, G.A.W.Y. and Abdel-Wahab, Y.H.A., 2003. The effects of traditional antidiabetic plants on in vitro glucose diffusion. Nutrition research, 23(3), pp.413-424. 
33. Hoseini, S.M., Taheri Mirghaed, A. and Yousefi, M., 2019. Application of herbal anaesthetics in aquaculture. Reviews in Aquaculture, 11(3), pp.550-564.

34. Jain, M., Ganju, L., Katiyal, A., Padwad, Y., Mishra, K.P., Chanda, S., Karan, D., Yogendra, K.M.S. and Sawhney, R.C., 2008. Effect of Hippophae rhamnoides leaf extract against Dengue virus infection in human blood-derived macrophages. Phytomedicine, 15(10), pp.793-799.

35. Jia, R., Liu, B.L., Han, C., Huang, B. and Lei, J.L., 2016. Influence of Stocking Density on Growth Performance, Antioxidant Status, and Physiological Response of Juvenile Turbot, Scophthalmus Maximu, Reared in Land Based Recirculating Aquaculture System. Journal of the World Aquaculture Society, 47(4), pp.587-599.

36. Jian, J. and Wu, Z., 2004. Influences of traditional Chinese medicine on non-specific immunity of Jian carp (Cyprinus carpio var. Jian). Fish \& shellfish immunology, 16(2), pp.185-191.

37. Kaviarasan, S., Vijayalakshmi, K. and Anuradha, C.V., 2004. Polyphenol-rich extract of fenugreek seeds protect erythrocytes from oxidative damage. Plant Foods for Human Nutrition, 59(4), pp.143-147.

38. Kawai, S., 1973. Studies on digestive enzymes of fishes-III. Development of digestive enzyme of rainbow trout after hatching and the effect of dietary change on the activities of digestive enzyme in the juvenile stage. Bull. Jpn. Soc. Sci. Fish., 39, pp.819-827.

39. Kirk, P.L., 1950. Kjeldahl method for total nitrogen. Analytical chemistry, 22(2), pp.354-358.

40. Kolarovic, J., Popovic, M., Mikov, M., Mitic, R. and Gvozdenovic, L., 2009. Protective effects of celery juice in treatments with doxorubicin. Molecules, 14(4), pp.1627-1638.

41. Krogdahl, Å., Hemre, G.I. and Mommsen, T.P., 2005. Carbohydrates in fish nutrition: digestion and absorption in postlarval stages. Aquaculture nutrition, 11(2), pp.103-122.

42. Laribi, B., Kouki, K., M'Hamdi, M. and Bettaieb, T., 2015. Coriander (Coriandrum sativum L.) and its bioactive constituents. Fitoterapia, 103, pp.9-26.

43. Lasram, S., Zemni, H., Hamdi, Z., Chenenaoui, S., Houissa, H., Tounsi, M.S. and Ghorbel, A., 2019. Antifungal and antiaflatoxinogenic activities of Carum carvi L., Coriandrum sativum L. seed essential oils and their major terpene component against Aspergillus flavus. Industrial crops and products, 134, pp.11-18.

44. Li, P., Jia, J., Zhang, D., Xie, J., Xu, X. and Wei, D., 2014. In vitro and in vivo antioxidant activities of a flavonoid isolated from celery (Apium graveolens L. var. dulce). Food \& function, 5(1), pp.50-56.

45. Lin, H., Tan, X., Zhou, C., Niu, J., Xia, D., Huang, Z., Wang, J. and Wang, Y., 2015. Effect of dietary arginine levels on the growth performance, feed utilization, non-specific immune response and disease resistance of juvenile golden pompano Trachinotus ovatus. Aquaculture, 437, pp.382-389.

46. Liu, F., Belding, R., Usategui-gomez, M. and Reynoso, G., 1981. Immunochemical determination of LDH-1. American journal of clinical pathology, 75(5), pp.701-707.

47. Lowry, O.H., Rosebrough, N.J., Farr, A.L. and Randall, R.J., 1951. Protein measurement with the Folin phenol reagent. Journal of biological chemistry, 193, pp.265-275.

48. Mansour, E.H. and El-Adawy, T.A., 1994. Nutritional potential and functional properties of heat-treated and germinated fenugreek seeds. LWT-Food Science and Technology, 27(6), pp.568-572. 
49. Mehboob, A., Khan, N., Atiq, U., Iqbal, K.J., Tayyab, R., Batool, S.S., Batool, H.S., Amjad, S. and Tanveer, M., 2017. Effect of Fenugreek as a Feed Additive on the Growth, Body Composition and Apparent Nutrients Digestibility of Striped Catfish Pangasius hypophthalmus Fry. Pakistan Journal of Zoology, 49(6).

50. Misra, H.P. and Fridovich, I., 1972. The role of superoxide anion in the autoxidation of epinephrine and a simple assay for superoxide dismutase. Journal of Biological chemistry, 247(10), pp.3170-3175.

51. Mittler, R., 2002. Oxidative stress, antioxidants and stress tolerance. Trends in plant science. 7(9), 405-410.

52. Modaresi, M. and Ghalamkari, G., 2012. The effect of celery (Apium graveolens) extract on the reproductive hormones. APCBEE Procedia, 4, pp.99-104.

53. Mohamed, G.A., Amhamed, I.D., Almabrok, A.A., Barka, A.B.A., Bilen, S. and Elbeshti, R.T., 2018. Effect of celery (Apium graveolens) extract on the growth, haematology, immune response and digestive enzyme activity of common carp (Cyprinus carpio). Marine Science and Technology Bulletin, 7(2), pp.51-59.

54. Montoya-Mejía, M., García-Ulloa, M., Hernández-Llamas, A., Nolasco-Soria, H. and RodríguezGonzález, H., 2017. Digestibility, growth, blood chemistry, and enzyme activity of juvenile Oreochromis niloticus fed isocaloric diets containing animal and plant byproducts. Revista Brasileira de Zootecnia, 46, pp.873-882.

55. Moriarty, D.J.W., 1973. The physiology of digestion of blue-green algae in the cichlid fish, Tilapia nilotica. Journal of Zoology, 171(1), pp.25-39.

56. Nadeem, M., Anjum, F.M., Khan, M.I., Tehseen, S., El-Ghorab, A. and Sultan, J.I., 2013. Nutritional and medicinal aspects of coriander (Coriandrum sativum L.): A review. British Food Journal.

57. Nagase, G., 1964. Contribution to the physiology of digestion in Tilapia mossambica Peters: Digestive enzymes and the effects of diets on their activity. Zeitschrift für vergleichende Physiologie, 49(3), pp.270-284.

58. Nelson, D.A., Morris, M.W., 1989. Basic methodology. Chapter 27. Hematology and coagulation, part IV: Clinical diagnosis and management by laboratory methods. Nelson, D.A., Henry, J.B., Henry, J.B, W.B. Saunder Company., Philadelphia, U.S.A. 578-625.

59. Nicolae Carmen Georgeta, Ișfan Nicoleta, Bahaciu Gratziela Victoria, Marin Monica Paula, Moga Liliana Mihaela, 2016. Case study in traceability and consumer's choices on fish and fishery products. AgroLife Scientific Journal, Vol.5, number 2, p.103-107

60. Oddepally, R., Sriram, G. and Guruprasad, L., 2013. Purification and characterization of a stable Kunitz trypsin inhibitor from Trigonella foenum-graecum (Fenugreek) seeds. Phytochemistry, 96, pp.26-36.

61. Pallavi, H. and Rajender, O., 2021. Role of disulfide linkages in structure and activity of Kunitz trypsin inhibitor from Trigonella foenum-graecum (Fenugreek) seeds and its porphyrin binding studies. Journal of Plant Biochemistry and Biotechnology, pp.1-10.

62. Pandeya, K.B., Tripathi, I.P., Mishra, M.K., Dwivedi, N., Pardhi, Y., Kamal, A., Gupta, P., Dwivedi, N. and Mishra, C., 2013. A critical review on traditional herbal drugs: An emerging alternative drug for 
diabetes.

63. Raissy, M., Ghafarifarsani, H., Hoseinifar, S.H., El-Haroun, E.R., Naserabad, S.S. and Van Doan, H., 2021. The effect of dietary combined herbs extracts (oak acorn, coriander, and common mallow) on growth, digestive enzymes, antioxidant and immune response, and resistance against $A$. hydrophila infection in common carp, Cyprinus carpio. Aquaculture, p.737287.

64. Rao, Y.V., Das, B.K., Jyotyrmayee, P. and Chakrabarti, R., 2006. Effect of Achyranthes aspera on the immunity and survival of Labeo rohita infected with Aeromonas hydrophila. Fish \& Shellfish Immunology, 20(3), pp.263-273.

65. Rusia, V. and Sood, S.K., 1992. Routine hematological tests. Medical laboratory technology, 1, pp.252-258.

66. Sahu, S., Das, B.K., Pradhan, J., Mohapatra, B.C., Mishra, B.K. and Sarangi, N., 2007. Effect of Magnifera indica kernel as a feed additive on immunity and resistance to Aeromonas hydrophila in Labeo rohita fingerlings. Fish \& shellfish immunology, 23(1), pp.109-118.

67. Sayeed, I., Parvez, S., Pandey, S., Bin-Hafeez, B., Haque, R. and Raisuddin, S., 2003. Oxidative stress biomarkers of exposure to deltamethrin in freshwater fish, Channa punctatus Bloch. Ecotoxicology and environmental safety, 56(2), pp.295-301.

68. Secombes, C.J., 1996. The nonspecific immune system: cellular defenses. The fish immune system: organism, pathogen and environment, 15, pp.63-103.

69. Shihabi, Z.K. and Bishop, C., 1971. Simplified turbidimetric assay for lipase activity. Clinical chemistry, 17(12), pp.1150-1153.

70. Silva De, S.S. and Anderson, T.A., 1994. Fish nutrition in aquaculture (Vol. 1). Springer Science \& Business Media.

71. Somogyi, M., 1952. Notes on sugar determination. Journal of biological chemistry, 195(1), pp.19-23.

72. Sowbhagya, H.B., 2014. Chemistry, technology, and nutraceutical functions of celery (Apium graveolens L.): an overview. Critical reviews in food science and nutrition, 54(3), pp.389-398.

73. Sunde, J., Taranger, G.L. and Rungruangsak-Torrissen, K., 2001. Digestive protease activities and free amino acids in white muscle as indicators for feed conversion efficiency and growth rate in Atlantic salmon (Salmo salar L.). Fish Physiology and Biochemistry, 25(4), pp.335-345.

74. Takahara, S., Hamilton, H.B., Neel, J.V., Kobara, T.Y., Ogura, Y. and Nishimura, E.T., 1960. Hypocatalasemia: a new genetic carrier state. The Journal of Clinical Investigation, 39(4), pp.610619.

75. Talpur, A.D. and Ikhwanuddin, M., 2013. Azadirachta indica (neem) leaf dietary effects on the immunity response and disease resistance of Asian seabass, Lates calcarifer challenged with Vibrio harveyi. Fish \& shellfish immunology, 34(1), pp.254-264.

76. Trinder, P., 1969. Determination of blood glucose using 4-amino phenazone as oxygen acceptor. Journal of clinical pathology, 22(2), p.246.

77. US Department of Agriculture. Agricultural Research Service, 2012. USDA Nutrient Database for Standard Reference. US Department of Agriculture, Agricultural Research Service. 
78. Van Hai, N., 2015. The use of medicinal plants as immunostimulants in aquaculture: A review. Aquaculture, 446, pp.88-96.

79. Wang, Z., Dong, J., Liu, L., Zhu, G. and Liu, C., 2013. Screening of phosphate-removing substrates for use in constructed wetlands treating swine wastewater. Ecological Engineering, 54, pp.57-65.

80. White, F.D. and Bowman, J.M., 1947. The estimation of trypsin. Canadian journal of research, 25(4), pp.153-161.

81. Wintrobe, M.M., 1934. Anemia: classification and treatment on the basis of differences in the average volume and hemoglobin content of the red corpuscles. Archives of internal medicine, 54(2), pp.256280.

82. Yang, S., Yan, T., Wu, H., Xiao, Q., Fu, H. M., Luo, J., Li, S. J., 2017. Acute hypoxic stress: effect on blood parameters, antioxidant enzymes, and expression of HIF-1alpha and GLUT-1 genes in largemouth bass (Micropterus salmoides). Fish \& shellfish immunology, 67, 449-458.

83. Zhao, L., Cui, C., Liu, Q., Sun, J., He, K., Adam, A.A., Luo, J., Li, Z., Wang, Y. and Yang, S., 2020. Combined exposure to hypoxia and ammonia aggravated biological effects on glucose metabolism, oxidative stress, inflammation and apoptosis in largemouth bass (Micropterus salmoides). Aquatic Toxicology, 224, p.105514.

84. Zheng, Z.L., Tan, J.Y., Liu, H.Y., Zhou, X.H., Xiang, X. and Wang, K.Y., 2009. Evaluation of oregano essential oil (Origanum heracleoticum $\mathrm{L}$.) on growth, antioxidant effect and resistance against Aeromonas hydrophila in channel catfish (Ictalurus punctatus). Aquaculture, 292(3-4), pp.214-218. 